\title{
ACTIONS, ObSERVATIONS, AND DECISION-MAKING: BIOLOGICALly INSPIRED STRATEgIES FOR AUTONOMOUS AERIAL VEHICLES
}

\author{
Greg Pisanich \\ Corey Ippolito \\ Laura Plice \\ gp@email.arc.nasa_gov \\ QSS Group Inc. \\ Computational Sciences Division
}

\author{
Larry A. Young ${ }^{1}$ \\ Benton Lau \\ larrv a.voungonasa gov \\ Amy NASA Rotorcraft Division
}

\author{
NASA Ames Research Center \\ Moffett Field, CA
}

\begin{abstract}
This paper details the development and demonstration of an autonomous aerial vehicle embodying search and find mission planning and execution strategies inspired by foraging behaviors found in biology. It begins by describing key characteristics required by an aerial explorer to support science and planetary exploration goals, and illustrates these through a hypothetical mission profile. It next outlines a conceptual bioinspired search and find autonomy architecture that implements observations, decisions, and actions through an "ecology" of producer, consumer, and decomposer agents. Moving from concepts to development activities, it then presents the results of mission representative UAV aerial surveys at a Mars analog site. It next describes hardware and software enhancements made to a commercial small fixed-wing UAV system, which include a new development architecture that also provides hardware in the loop simulation capability. After presenting the results of simulated and actual flights of bioinspired flight algorithms, it concludes with a discussion of future development to include an expansion of system capabilities and field science support.
\end{abstract}

\section{INTRODUCTION}

Autonomous aerial vehicles are poised to revolutionize aeronautics. New missions and applications are being defined and demonstrated at an accelerating pace. Vehicle autonomy will ultimately drive aerial vehicle design, leading to radically new vehicle configurations and concepts that no longer have to be constrained to the limitations of manned flight. Further, many of these emerging applications will redefine the classic perception of aerial vehicles into one that sees these

${ }^{1}$ Senior member, Aerospace Engineer vehicles as being highly mobile robots capable of threedimensional access ${ }^{1-3}$.

One new emerging application for aerial flyers is their potential use in planetary science missions. In order to find acceptable utility in a planetary science role, though, it will be essential to develop robust, computationally efficient, and mission-effective means of effecting autonomous flight/mission control of these future planetary aerial vehicles, or "aerial explorers." This is the fundamental goal of the NASA "BEES for Mars" project".

This paper builds upon preliminary work from the "BEES for Mars" project investigating the feasibility of defining and implementing bio-inspired flight/mission behaviors for autonomous aerial vehicles, acting as surrogate demonstrator platforms for aerial explorers for future Mars robotic science missions ${ }^{5-6}$. As an offshoot of that initial effort, the "Intelligent Aerial Vehicle (IAV)" project has made significant progress towards employing bio-inspiration in developing aerial explorer mission scenarios and autonomy architecture concepts.

Bio-inspiration can be a powerful tool when applied to engineering problems ${ }^{7}$, particularly the development of intelligent systems. Animals and Man can be described as organisms that operate under a simple behaviorai model. They are motivated to do Acitions, because of the changing state of their emotions and what they sense, or Observations they make, in the world.

In this paper, we describe how the thoughts -- or Decision-Making -- and actions of animals can be described using a behavioral model. We then describe how a similar model can be applied to autonomous uninhabited aerial vehicle (AUAV) operation, and how inspiration from Nature can yield new approaches to AUAV search and find strategies. 


\section{Previous Work}

Preliminary work on the IAV effort focused on the definition of bio-inspired mission concepts and flight "behaviors" that would successfully effect Mars aerial explorer demonstrations with terrestrial surrogate vehicles.

Mission concepts ${ }^{5-6}$ derive from situations in the biological world that are oriented toward "search and find" requirements: a mission using dropped aerial probes based on dissemination and survivorship curves, and a terrain-influenced search trajectory derived from predation strategies. A small compendium of bioinspired behaviors was defined for an aerial vehicle. These behaviors were categorized into general groups: UAV "primitive" tasks, actions, observations, and planning/decision-making. The use of stochastic search strategies was also emphasized in this preliminary work.

Figure 1 illustrates an early IAV implementation demonstrating conventional search and find behaviors. The demonstration used a 2-meter wingspan UAV system (Fig. 2a.) that had been enhanced to include a ground based adaptive decision component. This decision component allowed the aircraft command sequence to be altered based on images that were recognized from video downlinked from the aircraft.

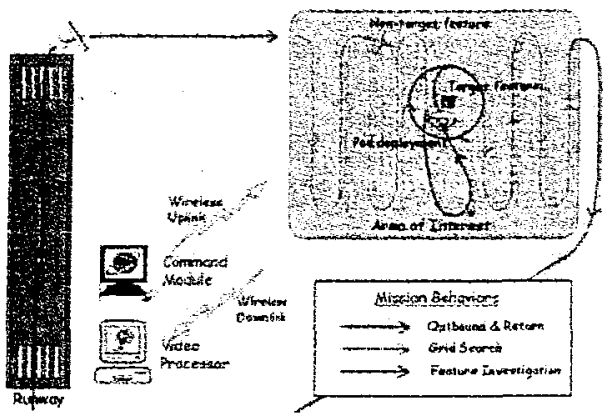

Fig i. Eariy UA V \& Drop Probe Demonstration.

The mission entailed searching for a target (in this case a large orange tarp on the ground, Fig. 2b.), visually recognizing the target, and dropping a small aerial imaging probe at/onto the position of the target, before resuming its search.

We conducted a series of UAV flights at the NASA Haughton Mars project on Devon Island, Nunavut, Canada. On this initial visit, video and flight data were gathered from several UAV flights. We also gained experience with logistics, operational limitations, and the numerous science opportunities associated with the Mars analog site. This experience proved to be invaluable for follow-on technology and field-science demonstrations at Haughton.
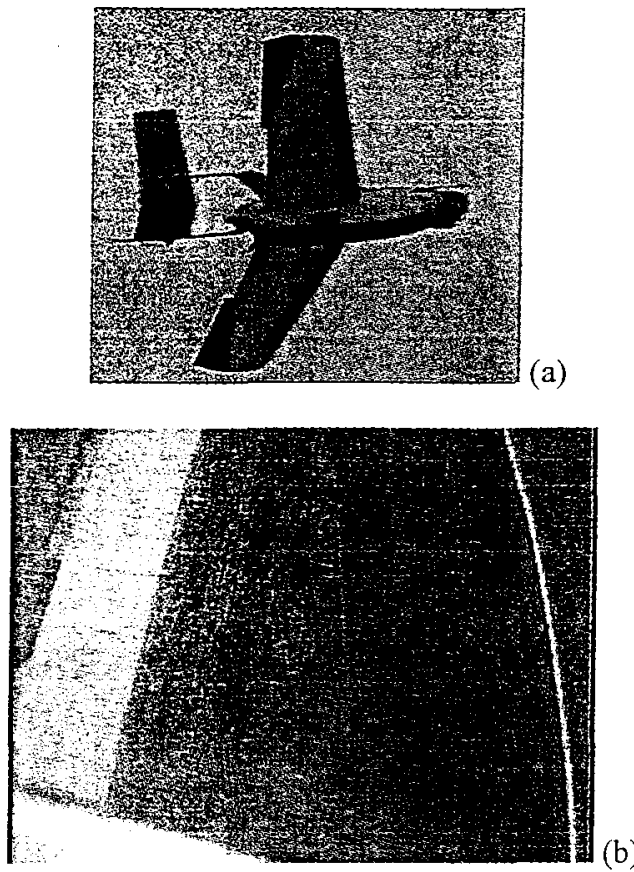

Fig 2. (a) MLB BAT UAV, and (b) Aerial View of Target ("Orange Tarp").

\section{AERIAL EXPLORERS: MORE THAN IMAGING FROM ON-HIGH}

In general, prior proposals for Mars flyer missions have envisioned these vehicles primarily as an imaging platform. The goal of a Mars flyer, then, has been to maximize the overflight area covered, taking photos as it went, until it inevitably crash landed. Two general approaches have been proposed for the launch of the flyer: air-deployment during entry and descent, or ballistic launch from a lander or rover. The goal of a flyer that would be shot out from a lander or rover might be to peer over the horizon at terrain that might be encountered subsequently on a traverse. These applications of Mars aerial vehicles view the vehicles as a sensor platform that was an extension of another system (get up high, stay up as long as you can, look down, and tell me what you see).

2

American Institute of Aeronautics and Astronautics 
Our vision for an aerial explorer on Mars is an Aerobot $^{3}$ that would consist of an aerial platform, science sensors (onboard and deployable), an intelligent system (that can make decisions on its own based on sensor input), communications (networked to other subordinate, coequal, or lead systems or aircraft), and a sophisticated mission profile and goals (that would allow it to make real time decisions to reconfigure flight goals and reallocate resources based on data measurements).

In this project, we are striving to maintain balance between two development goals. The first is that we are working to develop an aerial explorer whose primary purpose is to maximize science return. But, second, we must also keep in mind that systems such as this will need to operate in a planetary environment, with different operational requirements. The next paragraphs discuss characteristics of each of these goals.

\section{Aerial Explorers for Science Mission}

UAVs optimized for science return (particularly for NASA science missions) will likely have different vehicle configurations and mission goals than conventional UAVS. These aerial explorers must contain imaging (video or photographic) and sensing capabilities (spectroscopy, atmosphere sampling) that may be similar to some terrestrial applications (such as military UAVs that may be looking for inorganic objects in an organic world). But high altitude sensing is a necessary but not sufficient functionality for sciehce capture. "Our vision of an aerial explorer must also be able to perform close-in investigation of areas of interest, to include low altitude flight, drop pods, and other deployable sensors.

We are working toward a notion of field science, an activity where a scientist is interacting dynamically with the environment. With access to geologists and life scientists at NASA Ames, our challenge is to work in conjunction with them to use UAVs to solve their science goals. Understanding their methodology will help us to better enhance the UAV (sensors, programming, and mission design). This improved understanding of the field scientist's methods should also allow this knowledge to eventually be codified and integrated into vehicle autonomy development.

\section{Aerial Explorers for Planetary Missions}

Aerial vehicles developed for planetary missions (for example, Mars, Titan, Jupiter, and Venus $^{2}$ ) must necessarily embody radically different design configurations than those for terrestrial UAVs. These aircraft design issues are beyond the scope of our work. Nonetheless, despite the physical differences between planetary (and terrestrial) aerial vehicles, they will have many common autonomy challenges.

Because of communication time lag (for example, several minutes one way to/from Mars), planetary aerial explorers must be fully autonomous. They will not have the ability to wait a solar day for Earth-based decision-making or an operator in the flight control loop. Planetary UAVS will also have other limitations. They will have limited flight endurance (15 minutes to 3 hours, by most estimates), no opportunity to refuel, limited landing opportunities, and little chance of multiple flights. Even with satellite data and advance planning, they may have limited prior knowledge of the area over which they must fly.

These limitations will drive the design of the platform, sensors, and the overall mission. With no requirement to return and land, planetary UAVs may be designed to fly missions that are broader in scope, than terrestrial applications. Once an area of interest is located, the aerial explorer may need to make decisions on resources available, against further exploration opportunities. Information returned by drop pods or other sensors will provide data to make these decisions. Finally, when the aerial explorer does terminate its flight, it should also have the capacity to safely carry instruments to the ground and provide added science retum. Where possible, the aerial explorer should also be able to choose that landing site in order to maximize these opportunities.

Gaining operational experience with UAVs in planetary analog sites such as Haughton Crater, or Devil's Playground in Utah, should continue to provide us with a better understanding of what characteristics should be built into aerial explorers.

\section{A Notional Mars Aerial Explorer Mission Profile}

By working with terrestrial field, and planetary, scientists, we are working to define potential mission profiles that continue to build upon the aerial explorer feature set. The following is an example of a mission description for an aerial explorer that would be tasked to search for water outflow patterns on Mars. These outflows, that some scientists theorize may still be active on Martian hillsides (possibly due to seasonal snow-melt), are prime candidates for investigation as they may provide important clues in the search for past, or still existent, life (Fig. 3).

American Institute of Aeronautics and Astronautics 


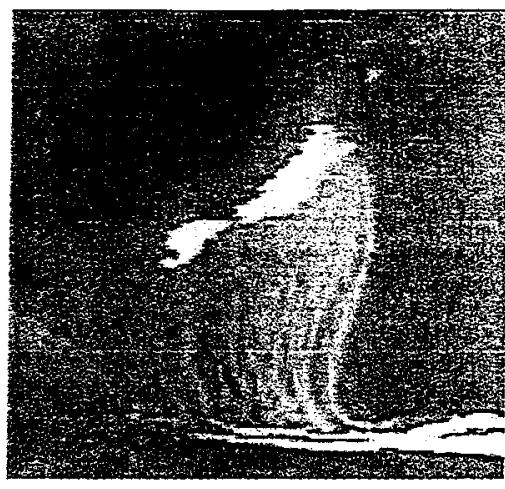

Figure 3. UAV photo of hillside snowmelt wateroutflow at Devon Island

In such a hypothetical mission scenario, an aerial explorer is deployed from an entry vehicle and descends via parachute to a high search altitude $(\sim 10,000 \mathrm{ft})$. It separates from the parachute and gathers a panoramic image and long range sensing data of the area around it (terrain, spectroscopy). Upon analysis, areas of interest are prioritized (flat areas with nearby hills, areas showing better spectral results) and the explorer heads in the direction of the most promising sectors. At each decision point in the mission, the decision, the data supporting it, and subsequent plans made by the explorer would be transmitted back to mission control via satellite relay.

As the explorer descends to an intermediate analysis altitude $(\sim 2000 \mathrm{ft})$ it begins a random behavioral search of the first area, looking for image (image recognitiois, rock distribution) and spectral (soil and rock composition) evidence of riverbeds. The explorer continually monitors resource constraints, (fuel, power and time) and decide whether to prolong a search in a particular sector or direction, based on the state of the science return.

Upon sensing high evidence of outflows, the explorer would commit to an area and descend to a low sensing altitude $(-500 \mathrm{ft})$. It would then use a combination of data sources (slope foliowing, rock dispersal distributions, linear feature following) to lead the explorer toward the potential source of the outflow. Drop pods would be dispersed over these areas to gather additional information on rock material, contact sensing, and visuals of flows at or near ground level.

As a potential source of outflows is identified (through spectroscopy, image, and terrain clues) the aerial explorer would extensively map the local area using its sensors through a series of circling and direct approach maneuvers. Drop pods containing tethered elements (i.e. "tetherbots") that would drape/catch on the hillside, as they float down, and return data for an extended time would also be deployed as close as possible to the outflow area. The result would be a three-dimensional data map of that site.

If the explorer had sufficient resources remaining, it could move on to another site. With low reserves, it would make the decision to terminate its flight in this area. In addition to the drop pods, the aerial explorer would also contain contact sensors on the nose, wing and fuselage of the aircraft that could survive impact with the terrain. By setting up an approach and impacting as close as possible to the outflow area, the aerial explorer would provide additional data as long as power was available. This "survivable crash" would also address on of the key problems identified for Mars flyers: namely it would provide adequate time for uplink/transfer data from the aerial vehicle to satellites and/or directly to mission control. Most projected Mars flyer flight times are of too short of duration to in-flight transmit gigabytes of imagery and other large data sets.

\section{Project DeVelopMeNT Philosophy}

There is obviously a large technology gap between the scenario described in the previous section and what is possible today. Our work is focused on developing technologies that will help bridge this gap. Our philosophy is grounded in a process of concept development, simulation, local flight tests and field demonstrations. This strategy is not unique, but works well for our applications.

Our development efforts on this project have focused on the creation of biologically inspired flight algorithms and mission concepts. These have been developed through consultations with domain experts such as biologists and planetary scientists. The results of which have been algorithms and mission scenarios.

We have developed a simulation environment and architecture (the "Reflection" architecture, described later in this paper) that allows us to program these algorithms and mission scenarios into a simulation of the aerial explorer, and the environment, and test fly the resulting mission. As this architecture also allows the software to be run on the flight hardware, further checks can be made on the flight readiness of the system.

As NASA Ames is co-located with the Moffett Federal Airfield, we have the opportunity to test the resulting 4

American Institute of Aeronautics and Astronautics 
flight code on our UAV. Some of the advantages to flying at Moffett Field are that we can fly often and the airfield provides clearance from other aircraft and RC frequency control. These are great advantages to our work. Some disadvantages of working on an operating airfield are that we have to work in parallel with manned flights within an airspace that can limit the mission scenarios and maneuvers we can attempt. The limited topography that the airport presents can be overcome by providing the aircraft with simulated terrain and sensor data. There are additional limitations such as scheduling flight times with other activities, and the very dense frequency congestion in the Silicon Valley, but these limitations are overshadowed by the ability to fly test fly our work often.

The field demonstrations are the ultimate evaluation of our vehicle autonomy work. Our goal is to test these mission scenarios and algorithms in scientifically interesting analogs of Mars terrain (Mars-analogs). Our intent is work with scientists that are familiar with the sites and have specific science return goals in mind. Some challenges to working at field sites include the logistics involved in getting to these often remote sites, flying within terrain constraints, and working with any airspace or frequency limitations. All of these factors are manageable compared to the valuable lessonslearned by flying these aircraft in "actual". conditions.

In working on the creation of mission concepts for Mars flyers we have set limitations to the scope of our effort that have helped maintain satisfactory progress in our domain. The first is that we currently expend limited effort into the development of sensors and sensor software and focus primarily on the flight and mission aspects of the problem. We use surrogate sensors (such as the visual recognition of a large orange tarp) and simulations as stand-ins for the lightweight optical/spectral sensors that are still in development. By focusing and expanding on the flight and mission aspects, we will be ready when these sensors are ultimately available.

Secondly, although we are aware of the constraints and challenges of flight in the atmosphere of Mars, we are not focusing on the design of a Mars capable aircraft. We instead concentrate on enhancing and operating a small commercial fixed-wing UAV to meet our demonstration goals. When a more advanced design becomes available, we should be able to replicate its resource, sensor, and flight characteristics in our simulation and adjust our missions and algorithms to match that aircraft and goals.

\section{BIO-INSPIRATION \& A SEARCH AYD FLND ALTONOMY ARCHITECTURE FOR AERLAL VEHICLES}

In our previous work ${ }^{5}$, we described how natural behavior (bio-inspiration) could be used and combined to achieve mission goals. In the following sections of this paper, we expand on this early work to discuss why the use of bio-inspired behaviors may be ideal for science and planetary missions. We also expand on our early "robotic ecology" work to describe a holiarchy architecture based on information.

\section{Search and Find for Planetary Science}

There are several reasons why conventional search algorithms, such as a grid search, or a search around a last known position, would not work well in a planetary science environment and biologically based algorithms may provide better performance.

The world and its extent may not be known at the onset. Information about the area may be sketchy at best (satellite maps, rough spectroscopy). With this state of knowledge, aerial exploration would most likely start a search in a promising direction. This method points to a directed random search with the capability of narrowing focus when one or more terrain "features of interest" are identified.

Goals are hard to define from the outset. A hypothesis is often put forth, but can be easily amended depending on what information is encountered. What was originally searched for mà not be found, or something totally unexpected -- but related to another hypothesis -- may be encountered.

Limited resources point toward covering larger regions rather than concentrating only on a small search. Rather than an exhaustive search in a single location, characterizing an area to some level and then moving on to additional sites could be preferable. As the explorer would probably not retum to previously visited sites, the decision-making employed to determine when to move to a new site/search becomes very important.

Scattered areas of interest may be located far from each other. separated by empty areas. Exhaustive search in this environment would quickly deplete resources. The need to look at the world from varying vantage points (high altitude with low sensor fidelity versus low altiude with high sensor fidelity) points toward the use of "fox and mouse" tacticss. 


\section{General Software Architecture "Economy of Nature" Inspired Model}

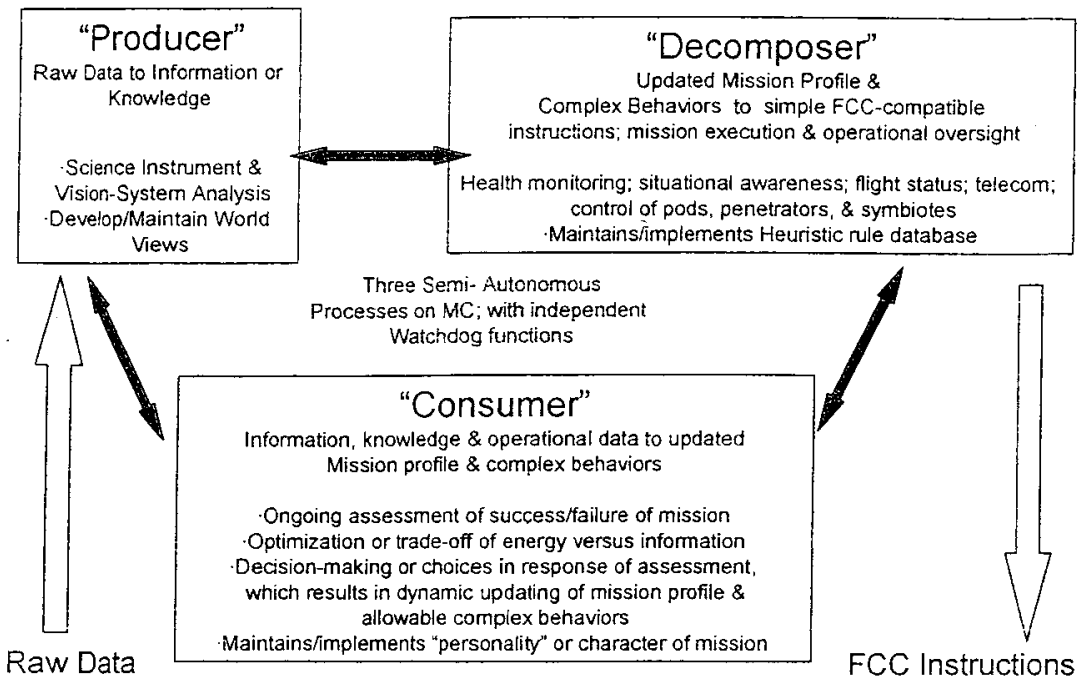

Fig. 4. Holarchy Conceptual Model of Aerial Vehicle

Natural marriage between bio-inspired search and find strategies and statistical random sampling theory. Rather than employing an exhaustive search of an area, a random sampling (by means of an intelligent random walk flight behavior, for example) of a statistically significant portion would yield data that could be predictive of the actual result.

\section{$\underline{\text { Robotic Ecology }}$}

We are working toward a general architecture for the development of autonomous air vehicles. Although this will initially involve the use of conventional programming and representation techniques, we are striving to move toward a behavioral architecture and programming model. In our current model, we use a producer, consumer and decomposer model borrowed from nature ${ }^{5-6}$ where the producer provides input (observations) into the system, the consumer evaluates the data and makes decisions about the input and the current state of the machine, and the decomposer translates those decisions into selections of actions that are applied to the world. Figure 4 shows a conceptual schematic of a holarchical, or "ecology," architecture as applied to a single aerial vehicle and its sub-systems. A holon is an individual member of the holarchy.

The description of a biological community is based primarily on the flow of energy. For a bio-inspired community of autonomous vehicles an analogy of energy and information is assumed needed to successfully model and sustain vehicle operation and execution of the planned mission(s). The gathering of information may be regarded as the motivating purpose for sending autonomous vehicles to Mars. The role of information producer can be filled by instruments and probes that collect information directly from the planetary surface or atmosphere. Information consumers take data from producers for processing and may release information in a different form to other information consumers.

Multiple information processing roles may be implemented onboard the same hardware platform. The steps in the information "food chain" show a similarity to the layers of abstraction familiar in the design of robotic software. Researchers on Earth may be the ultimate information decomposers about the Martian surface.

Table 1 is an illustrative example of a Mars robotic "ecology" employing aerial explorers. The large number of robotic systems in the ecology is unlikely for Mars missions in the near future. However, the large number and variety of robotic systems is entirely possible/feasible for field science testing at Mars analog sites. Such an ecology has already been mimicked ${ }^{9-10}$. 
Table 1. Examples of a Holarchical Taxonomy for Autonomous Aerial Vehicles.

\begin{tabular}{|c|c|c|c|}
\hline Design. & Description & $\begin{array}{l}\text { Raison } \\
\text { d'être }\end{array}$ & \begin{tabular}{|l} 
Ecology \\
Holarchical \\
Role(s) \\
\end{tabular} \\
\hline $\mathrm{AEI}$ & $\begin{array}{l}\text { Aerial Explorer } \\
\# 1\end{array}$ & $\begin{array}{l}\text { System; equal } \\
\text { relationship } \\
\text { with AE2, } \\
\text { AE3, \& } \\
\text { ROVER1; } \\
\text { subordinate to } \\
\text { BASE }\end{array}$ & $\begin{array}{l}\text { (At highest } \\
\text { level) Producer } \\
\& \text { Consumer (at } \\
\text { sub-level, a } \\
\text { collective of all } \\
\text { three) }\end{array}$ \\
\hline CAMIA & $\begin{array}{l}\text { Forward pointing } \\
\text { camera on AEI }\end{array}$ & $\begin{array}{l}\text { Subsystem; } \\
\text { subordinate to } \\
\text { AE1 }\end{array}$ & Producer \\
\hline CAMIB & $\begin{array}{l}\text { Downward } \\
\text { pointing carnera } \\
\text { on AEl }\end{array}$ & Subsystem & Producer \\
\hline CAMIC & $\begin{array}{l}\text { Pan/Tilt camera } \\
\text { with zoom on } \\
\text { AEl }\end{array}$ & Subsystem & $\begin{array}{l}\text { Producer \& } \\
\text { (Potentially } \\
\text { with pre- } \\
\text { processing } \\
\text { capability) } \\
\text { Decomposer }\end{array}$ \\
\hline $\mathrm{FCl}$ & $\begin{array}{l}\text { OEM UAV flight } \\
\text { computer \& } \\
\text { avionics system }\end{array}$ & Subsystem & Decomposer \\
\hline$\overline{\mathrm{DPI}}$ & $\begin{array}{l}\text { Drop Probe on } \\
\text { AEl }\end{array}$ & $\begin{array}{l}\text { Subsystem \& } \\
\text { (Potentially) } \\
\text { System } \\
\end{array}$ & $\begin{array}{l}\text { Producer \& } \\
\text { decomposer }\end{array}$ \\
\hline$\widehat{A E 2}$ & $\begin{array}{l}\text { Aerial Explorer } \\
\# 2 \text {; "expendable" } \\
\text { short range recon; } \\
\text { rover-launched } \\
\text { rocket glider }\end{array}$ & System & Producer \\
\hline AE3 & $\begin{array}{l}\text { Aerial explorer } \\
\# 3 ; \text { a "Mars } \\
\text { rotorcraft" }\end{array}$ & System & $\begin{array}{l}\text { Producer \& } \\
\text { Consumer }\end{array}$ \\
\hline MR3A & $\begin{array}{l}\text { Micro-rover; AE3 } \\
\text { ground deployed }\end{array}$ & System & $\begin{array}{l}\text { Producer \& } \\
\text { Consumer }\end{array}$ \\
\hline ROVERI & Rover \# I & System & $\begin{array}{l}\text { Producer \& } \\
\text { Consumer }\end{array}$ \\
\hline$\overline{B A S E}$ & $\begin{array}{l}\text { Base camp, or } \\
\text { mission control }\end{array}$ & $\begin{array}{l}\text { System; man } \\
\text { machine } \\
\text { interface }\end{array}$ & $\begin{array}{l}\text { (Uitimate) } \\
\text { Consumer }\end{array}$ \\
\hline
\end{tabular}

To fully define such a robotic ecology is necessary to define individual system/sub-system roles and relationships. Table 1 touches upon some of the roles that might be applicable for Mars exploration, entailing, in part, the use of aerial explorers. There are four possible relationships between individual systems/subsystems: independent/isolated (numeric value of 0 ); subordinate (0.5); "self" identification (1); equal (2); lead (3). For the systems with an "equal" relationship, there can be sub-classes of that relationship: cooperative (numeric value of 2.1); neutral (2.2); competing (2.3). These relationships between systems/subsystems, A, within the ecology hierarchy can be defined by a "relationship" matrix, $\mathbf{R}$, such that $\mathbf{A} \rightarrow \mathbf{R A}$. The matrix $\mathbf{R}$ is simply the "identity" matrix if systems/sub-systems acted in isolation from each other. An illustrative example of an interactive ecology of aerial explorers and other systems/subsystems (building off of Table 1) is

$$
\left[\begin{array}{c}
\text { AEl } \\
\text { CAMlA } \\
\text { CAMlB } \\
\vdots \\
\text { AE2 } \\
\vdots \\
\text { ROVERI }
\end{array}\right] \rightarrow\left[\begin{array}{ccccccc}
1 & 0.5 & 0.5 & \cdots & 2.1 & \cdots & 2.3 \\
3 & 1 & 0 & \cdots & 0 & \cdots & 0 \\
3 & 0 & 1 & \cdots & 0 & \cdots & 0 \\
\vdots & & & & & & \vdots \\
2.1 & 0 & 0 & \cdots & 1 & \cdots & 3 \\
\vdots & & & & & & \vdots \\
2.3 & 0 & 0 & \cdots & 0.5 & \cdots & 1
\end{array}\right]\left[\begin{array}{c}
\text { AE1 } \\
\text { CAMlA } \\
\text { CAMIB } \\
\vdots \\
\text { AE2 } \\
\vdots \\
\text { ROVERI }
\end{array}\right]
$$

These ecology roles and relationships can be initially prescribed, but more sophisticated modeling may allow them to evolve with time and information resources/dynamics. In this case the ecology interaction can be moderated by means of techniques for mathematical modeling of population dynamics from biology.

Observations/Producer In an intelligent air vehicle, observations may come from several sources. Some may come from the sensors themselves. These would include control sensors, video, temperature. Sensor data could be fused to provide additional information.

Observations could also come from state data, providing a type of memory for the system. This could involve trend data (aircraft is descending) or state change data (we have 2 pods remaining). Observations would also include decisions made and actions taken by the air vehicle. Observations, as they are updated would be made available to the decision-making system.

Decision-Making/Consumer The decisionmaking component would consume (evaluate) the data provided and decide whether the data observed was bringing the aircraft closer towards the flight goals.

This could be achieved through a conventional intelligent system architecture that would represent the goals and processing to achieve them as a conventional planner and execution system. The air vehicle would be controlled by predefined plans and conditional execution of routines based on the data and resources sensed. The output of the decision system would be a single or sequence of behaviors to be performed by the decomposer. Alternatively, an "emotional holon" decision-making process/methodology might be employed ${ }^{8}$ (refer to Fig. 6).

The following set of "emotions" could be proposed for the autonomous aerial vehicle conducting search and 
find scientific investigations: "fear" (i.e. real-time assessment of level of risk); "desire" (current probability of mission success); "confidence" (real-time assessment of system capability, e.g. amount of fuel remaining, number of onboard probes, etc.); "altruism" (relative assessment of individual versus group success; applicable to multiple robotic flyers/vehicles). This should be considered a minimum list of emotions necessary for autonomous UAV operations; there may well be others that could be implemented in. UAVs.

Effecting emotional modeling of aerial vehicle goes hand in hand with definition of motivations and personalities of the aggregate of producer, consumer, and decomposer agents that comprise the "ecology/economy of mind" that allow for the successful autonomous operation and decision-making required for a Mars aerial explorer ${ }^{6}$. Implementation of an aerial vehicle personality can work if at least four attributes are modeled (refer to Fig. 5):

$\begin{aligned} & \text { Passive } \rightarrow \text { Aggressive } \\ & \text { (or, rather, Energy Conservative) } \rightarrow \text { versus Energy Profligate) }\end{aligned}$
Risk Adverse $\rightarrow$ Risk Tolerant
(or, rather, Simple Sensing/Behaviors) $\rightarrow$ (versus Complex
Sensing/Behaviors)
Dissemination Conservative $\rightarrow$ Profligate
(r-stategy wrt pods/penetrators) $\rightarrow$ (versus K-strategy)
Social $\rightarrow$ Asocial
(Large exchange of information between "equal" systems) $\rightarrow$ (No exchange)

A relative numeric scaling ( $0-10$, for example) between these "personality" attributes allows the pre-flight tailoring of the vehicles' ultimate response to observations and actions during the flight. Finally, it is imperative that "search for water' and 'hunt for life' higher-level goals be heuristically expressed/defined in terms of simple observational rules and subsequent UAV actions. This also holds true for safety of flight and health monitoring. Some illustrative conditional rules are shown below.

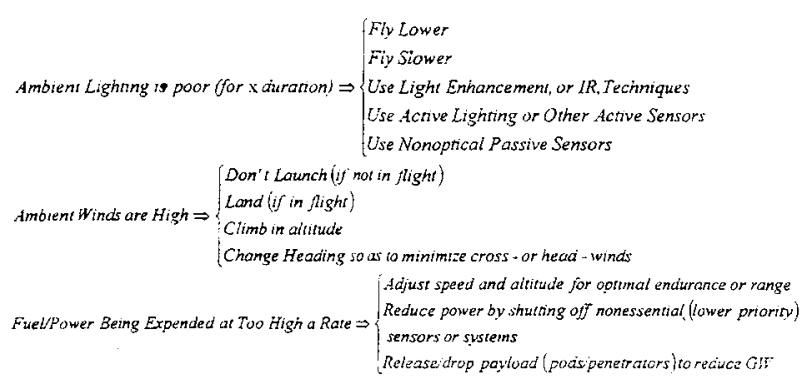

Actions/Decomposer The Decomposer would implement the actions called for by the Consumer component. These high level commands would be broken down by the aerial vehicle mission computer into "primitive" tasks that could be communicated to and executed by the OEM flight computer - as in the case of the small fixed-wing UAV used by the IAV project. Actions could involve changing the direction and altitude of the vehicle (behaviors), deploying sensor pods, reconfiguring the sensor system, adding additional processing steps to the observations, or sending observations back to the base.

Fusion of Concepts into an integrated strategy To arrive at an effective autonomous system implementation for the aerial vehicle demonstrator it is necessary to fuse all of the concepts noted so far into an integrated bio-inspired holarchy autonomy architecture. Figures 5 and 6 attempt to present a high level schematic of an integrated system example.

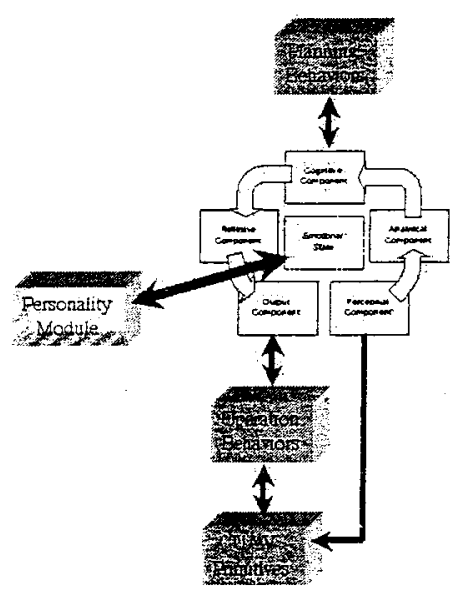

Fig. 5. Integrating Personality Attributes \& Behaviors to the Emotional Holon Schema.

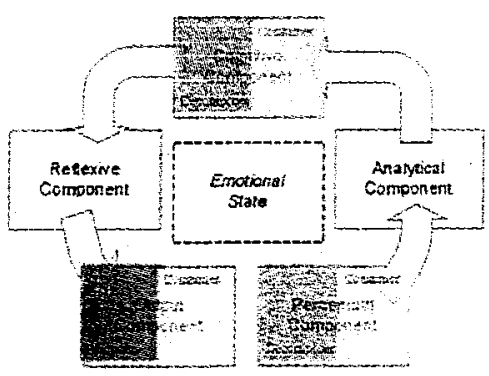

Fig. 6. Integrating "Economy/Ecology of Mind" Roles Into a Single, Individual Robotic System Holarchy 


\section{DEVELOPMENT ACTIVITLES}

We have so far examined concepts, possibilities and methodologies for aerial exploration. In the next sections, we discuss activities of field experiments, software and hardware development, and demonstration flights that were accomplished by our team in 2003.

\section{Field Experiments}

Mission-Representative Aerial Surveys. The IAV project, in order to refine its bio-inspired mission scenarios, invested a significant amount of effort conducting mission-representative aerial surveys at the Haughton Crater, Devon Island, Mars-analog site Figures 7 and 8 are two representative aerial survey image mosaics of the Devon Island site. Both sets of mosaics were taken during a single UAV flight along the outskirts of the "Von Braun Planitia"." Figure 7 shows a small valley (images taken with a downward pointing camera); valleys, and how they .form, are topics of intense interest to Mars planetary scientists. Figure 8 shows a ridgeline with prominent rock outcroppings.

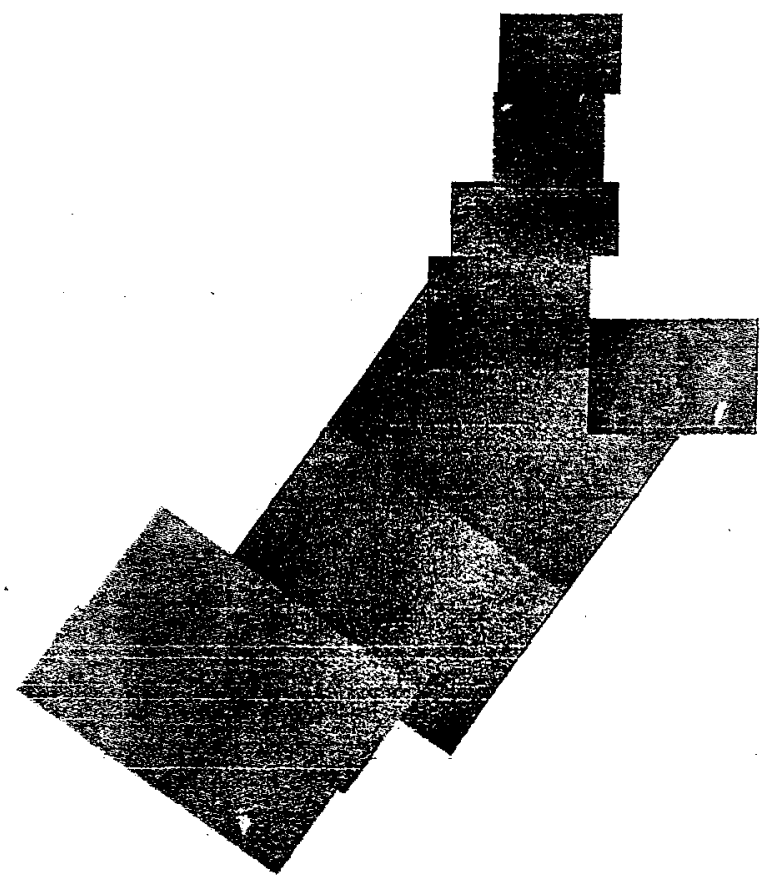

Fig. 7 Small Valley on the Outskirts of the Von Braun Planitia.

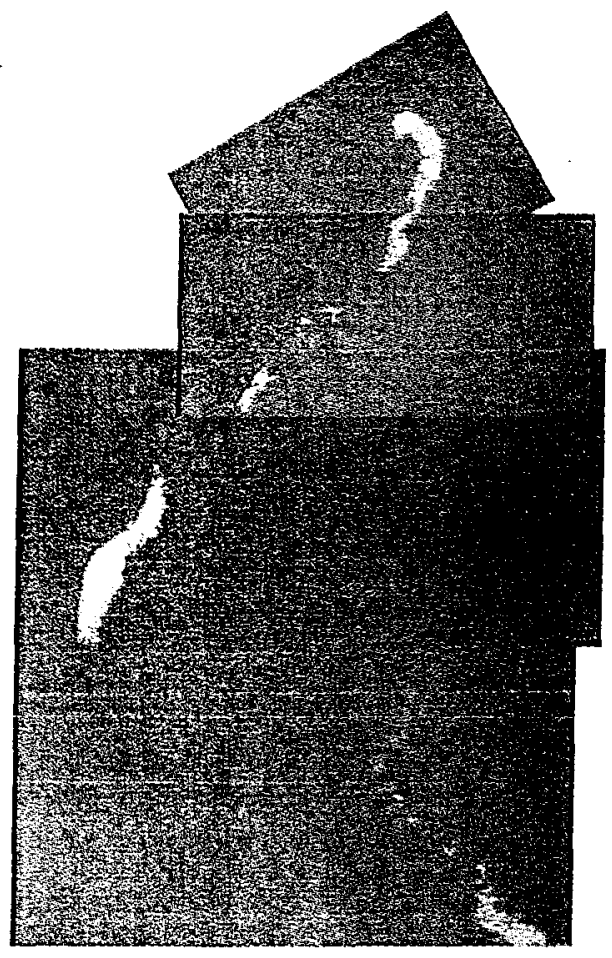

Fig.8 Ridge Line Rock Outcroppings

Drop Probe Release and Imaging We also made considerable progress towards demonstrating the potential of drop probes with imaging cameras, and other sensors, used in conjunction with aerial explorers. Testing was conducted at the NASA Ames Moffett Field airstrip as well as the Haughton Crater, Devon Island site (Fig. 9). The release of the drop probe has been fully automated to require only a rudimentary vision-system to recognize a target, redirect the UAV back to the location of the target, and release the drop probe over the target location.. This has been accomplished both with ground-based and onboard software. More sophisticated drop probes ${ }^{9-10}$ and UAV behavioral responses are planned for the future.

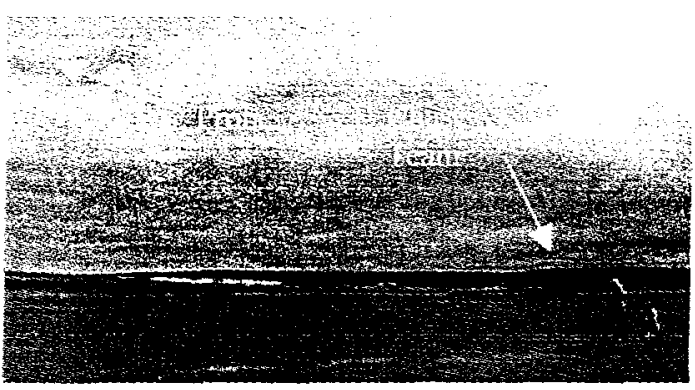

Fig. 9 Recovery of Drop Probe. 
Lessons Learned from Haughton We learned several important lessons from our field work and subsequent imagery analysis efforts that would not have been apparent if we hadn't visited this Mars analog site. First, in this area largely barren of life, the notion of our biologically inspired "fox and mouse" mission scenario, illustrated in our earlier paper, became more apparent. In the fox and mouse scenario, the fox avoids the mouse-barren fields and focuses his efforts on the tree line, where the mice venture forth from the briar patch. In viewing the video of our higher altitude flights, we were struck by the relatively long distances of empty plains punctuated by interesting riverbeds, valleys, and rock outcroppings. These were the areas of highest science interest.

Second the results from our higher altitude flights begged for additional flight behaviors. Seeing interesting features glide by on the video pointed out the need of circling flight to loiter over an area and look closer at objects. Also, seeing a hint of an interesting area along the edge of the video stream, that would not be over flown by the current flight path plan, pointed out the need for a gimbaled camera and the ability to alter the course in that direction based on the recognition of interesting objects.

\section{Hardware and Software Development}

Processor Integration Our initial development effort was focused on integrating an additional processor to the aircraft to support advanced reasoning and behaviors. This "mission computer" augrnents the OEM avionics "flight computer" onboard the UAV used for flight operations.

The flight system is built on an MLB "BAT" UAV outfitted with a PC/104 based CPU stack that interfaces through serial communication lines with the MLB flight computer and the onboard radio modem (Fig. 10). The $\mathrm{PC} / 104$ receives state information by monitoring telemetry information sent from the flight computer to the ground station, and uploads new flight plans to the flight computer. The PC/104 also receives video input from the onboard camera system that is used for image processing experiments. The $\mathrm{PC} / 104$ stack is powered from the UAV power supply and consists of a Digital Logical SmartCore 700MHz Pentium 3 CPU board, a DC/DC power supply board and a video capture board.
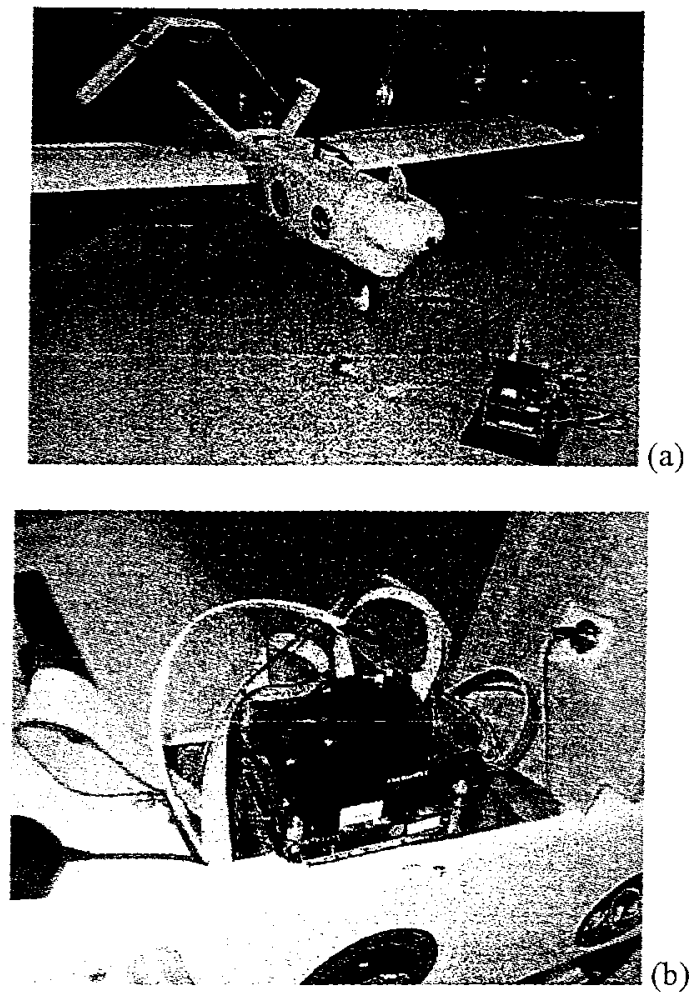

Fig 10. - PC-104 "Mission Computer": (a) outside of Small UAV and (b) partially installed.

The flight software on the $\mathrm{PC} / 104$ performs the highlevel mission planning and video processing for each experiment. The current implementation of the software is built on components that were tested in simulation, and consists of three main concurrently operating components. A data monitoring component monitors the serial port connections to the flight computer and the radio modem, relaying information back and forth between these two hardware components while maintaining the system state for other software components in the system by monitoring the telemetry data periodically by the flight computer. A second component processes video from the onboard camera system and triggers events that are received by the other software components. The third component performs the high level mission planning, receiving state information decoded from the data monitoring module and events from the video processing module, processing this information, and then uploading new flight plans to the onboard flight computer accordingly.

With the mission computer installed and flight tested, another key project milestone was completed with the port of the simple trigger (orange tarp) ground10

American Institute of Aeronautics and Astronautics 
computer-based image recognition software to that onboard computer. The vision-based target recognition and drop probe work completed in $2002^{5,9}$ was reproduced using the onboard system.

"Reflection" Architecture and Simulator In parallel with this effort, we have teamed with another UAV project at Ames to co-develop an architecture and hardware-in-the-loop simulation capability. This system allows us to quickly develop and test flight algorithms and software both in simulation and on the actual flight hardware. It also provides post flight capability to visualize and evaluate the flight performance of algorithms. Figure 11 shows a display interface from the simulator. Ultimately, we intend to implement the robotic ecology and emotional holon concepts noted earlier using this architecture.

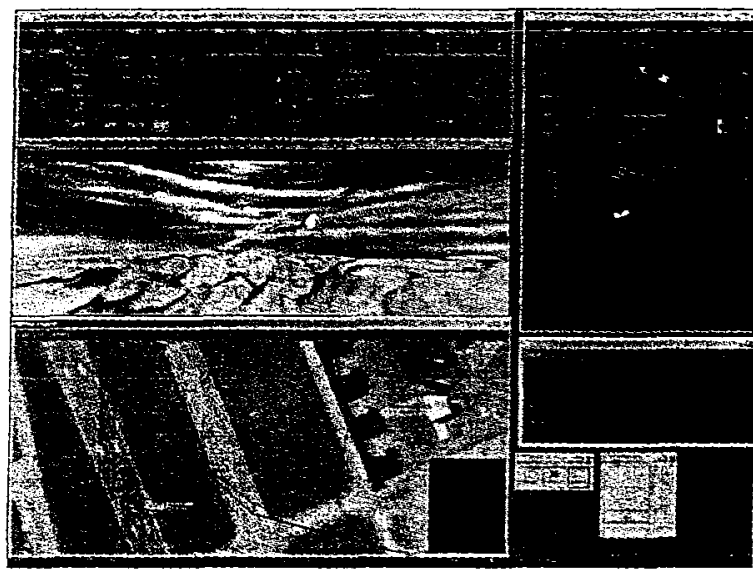

Fig 11. - Reflection Simulator Display Interface.

The Reflection Architecture is a component-based cross-platform object-oriented application framework for rapid development of high reliability simulations and real-time embedded systems. It operates by the principle of data reflection, where modular components operate in a larger system configuration on information that is gathered, filtered, and delivered to the component without ever interacting directly with other modules. Data reflection provides a layer of isolation that surrounds each component, allowing for a reusable and highly configurable framework. Modules operate on data completely within their own private context without having to consider where the data comes from or where it goes. This greatly reduces the code size and complexity for each module while freeing developers to focus entirely on implementing functionality rather than on larger architectural issues. The principle of data reflection also greatly simplifies the implementation of concurrency, allowing any module to take part in a multitasking system without the need to consider complex inter-module concurrency issues such as dead locks, race conditions and priority inversion.

This architecture also provides a rapid development capability that also supports rigorous testing of components and systems throughout development. The scripting functionality allows for multiple system configurations to be easily created during development, allowing development of automated regression tests, simulated scenario tests, stand-alone component tests, etc.

Traditional methods of testing are usually invasive to software and can increase code size and complexity, which are two factors that always degrade software quality. With the Reflection architecture, hundreds of testing configurations, scripts, scenario, etc., can be created without a single modification to source code, reducing the amount of resources required to thoroughly test components. Further, components can be swapped into and out of the architecture as needed, allowing for the system to support simulation testing of varying configurations at various levels of fidelity. For instance, control modules can be tested with simulated vehicle information in a UAV application by replacing sensor interface modules with other reusable modules implementing a simulated vehicle by changing a few lines in a script file.

The system also facilitates component reuse, as developers can selectively create configurations to test selected modules or the entire system by utilizing a growing repository of preexisting modules that include generic data visualization modules, vehicle simulations at various levels of fidelity, data signal generators, and interface modules to allow users to manipulate data with joysticks or other hardware. For simulation use, complete large-scale simulations for research or prototyping can be rapidly assembled by selecting data modules from this repository without writing a single line of code and with functionality customized to the project's requirements.

The Reflection simulator is composed of several flight simulation components that were assembled and rewritten to use the architecture. Visible in Figure 11 are moving map, scene visualization, Mode Control Panel, and Primary Flight Display components. Not shown, but critical to the simulation, are aircraft model, autopilot, and sensor components. 
We are also enhancing this software to support real time simulation of sensor data to the aircraft while in flight. This capability will allow us to simulate environments and sensor inputs that may be very different than the launch point or location in which the aircraft is being flown.

Work towards an emotional holon system The purpose of the emotional holon concept is to formulate and implement a system for controlling the behavior of a UAV that provides adaptive rational decision-making, learning, action selection, and reflexive behavioral control in achieving goals $s^{8}$.

The emotional holon theoretical model is largely built on the Pandemonium and Global Workspace theories. Emotional primitives, codelets, are represented as control theoretic transformations, with initial implementation consisting of proportional-derivate controllers. The activated codelets that are represented by the arena analogy in Pandemonium present the system with the active control blocks for the system. Through combination of emotional modeling theories and control theoretic formulation, we hope to show that emotional modeling can be an effective tool for designing and implementing adaptive control systems for aerial explorers.

\section{Terrestrial flight demonstration of behavioral flight} concepts.

We are in the process of completing a series of UAV flights at Moffeti Fieid, with the goal of denonstrating and evaluating the utility of several biologically-based flight behaviors that could be used by an aerial explorer. The mission modes and biologically-based behaviors chosen for these flights include:

1) High altitude preliminary search returning a panoramic view of the area. For this mode we have chosen to use a circling, overlapping flight path as exhibited by hawks or eagles when surveying an area.

2) Mid-level long-range search for an item over an area with little previous data. A randomly generated flight path, as used by bacteria, insects, or other animals searching a new area was selected for implementation.

3) Low-level search of an area where items have been previously identified. For this mode, a "Fox vs. Mouse" terrain terrain-following behavior was chosen.
The following sections compare simulation results of these behaviors to actual flight path data. Because we do not currently have access to a complete set of flight and autopilot design information on the MLB UAV, these plots contain several assumptions/approximations. The first is that the simulations were performed with using a "scaled" Cessna 172 aircraft simulation model flown at an altitude of 1000 feet and airspeed of 100 miles per hour. These simulation results were qualitatively scaled to match the UAV experimental results. The second is that results show that while the autopilot used in the simulation transitions to its next waypoint prior to reaching it, the autopilot on the UAV requires it to pass through a waypoint before transitioning. Finally, the simulated versus actual flight paths/behaviors compared were chosen to be representative of the data collected and are not correlated in time sequence.

Flight Behavior: Hawk Flight Our first experiment demonstrated the $\mathrm{PC} / 104$ commanding a circling pattern that drifted randomly over time to search an area. As the aircraft drifted the flight system computers processed the video input looking for a red target tarp on the ground. Figure 12 plots the ground path of the aircraft against the ground plot of a simulated aircraft with the same random algorithm. The UAV was commanded to fly at an altitude of $400 \mathrm{ft}$ AGL with an airspeed of 30 miles per hour.

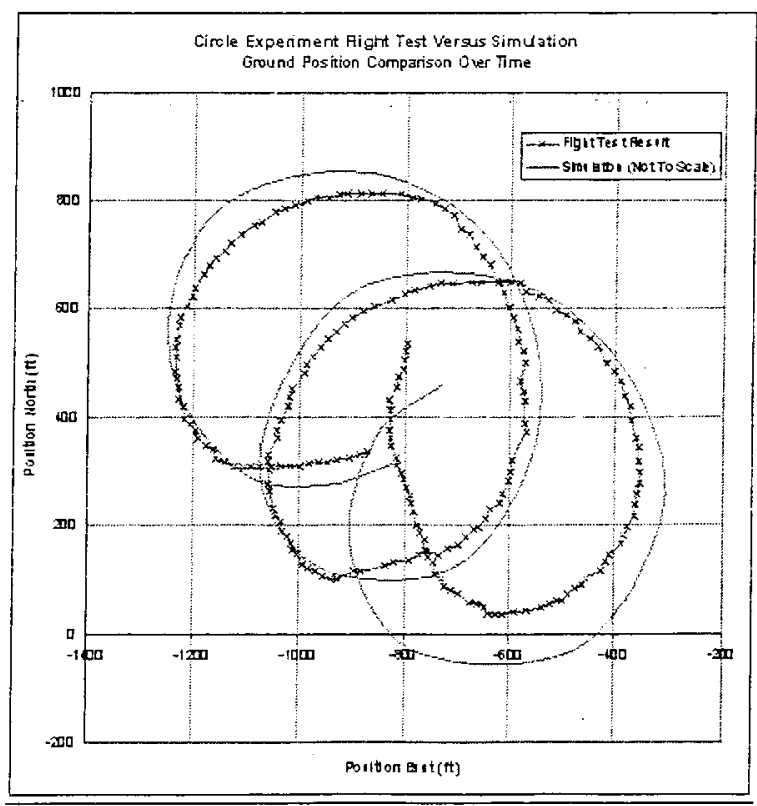

Fig. 12. Simulation vs. flight ground path of circling algorithm. 
Despite the simulation approximations noted, the agreement between the simulator and flight test results are quite good for the "Hawk" circling flight behavior. The random permutations to the circling behavior are clearly captured in Fig. 12, as well.

Flight Behavior: Random-Walk. Our second experiment implemented an ad hoc "intelligent" random-walk algorithm that plotted a random course over the search area starting from one boundary and continuing to the other boundary by computing random length legs and directions while attempting not to return to points previously searched. When a boundary was crossed, the algorithm would mirror the search direction about the boundary to keep the aircraft within the search area. The result of the algorithm is shown in Fig. 13, plotted against the actual UAV position and the simulation results when the aircraft was fed the same random course. Good agreement was found in this intelligent random-walk flight behavior.

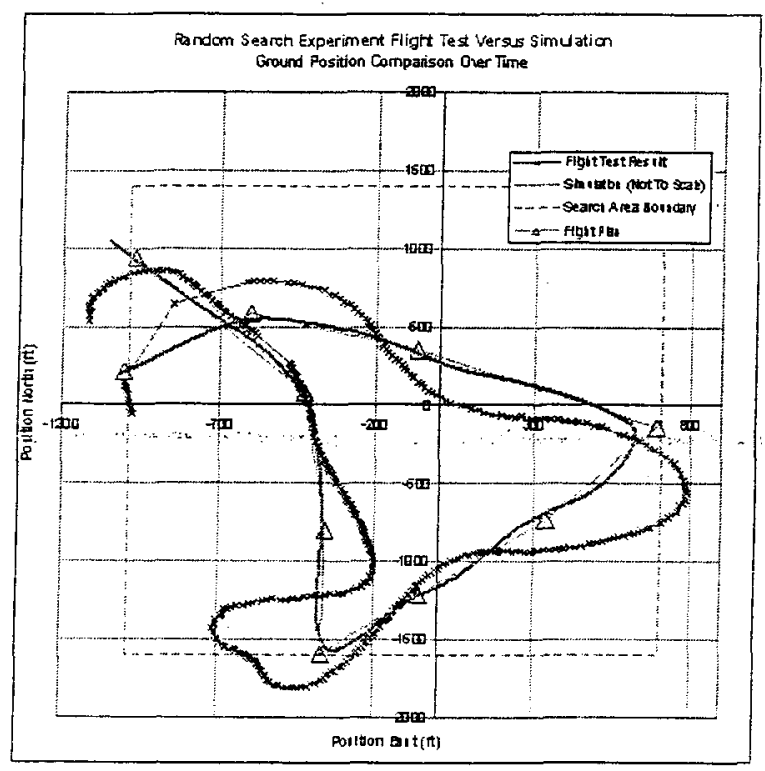

Fig. 13. Simulation vs. flight ground path of an intelligent random-walk flight algorithm.

Flight Behavior: Terrain Following. Our third experiment implemented a behavior capable of recognizing and following a low-lying region such as a ravine or gorge. A terrain following autopilot was created that accepts input from a ground elevation sensor, computes an estimated ground slope based on the sensor data history, and then computes a desired heading from this information using ad hoc estimation algorithms. The heading commands are directed into an under damped heading hold PID autopilot. The experiment was initially tested using circular and rectangular ground geometries. Figure 14 shows the results of a simulation of the aircraft finding and following a ground terrain geometry similar to a horseshoe gorge. The ground track of the aircraft is plotted against a gray scale terrain elevation map, where dark areas represent low-lying regions. Similar experiments have been run with ridgelines and crater rim terrains.

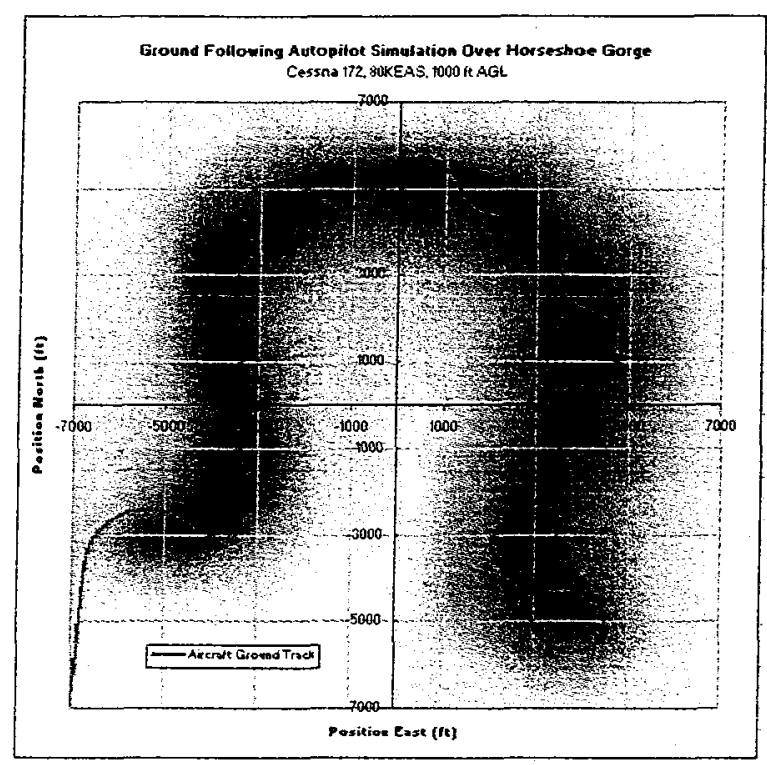

Fig. 14. Simulation ground path of a terrain following: flight algorithm.

\section{FUTURE WORK}

This past year we completed significant ground work towards an aerial explorer: a tested flight platform with on board and data linked processing capabilities, an enhanced software module development and hardware in the loop test capability, flight test results to include biologically-based behaviors, and field work in scientifically interesting areas that has included post imagery analysis.

\section{Expanding System Capabilities}

There are several directions in which we would like to expand this aerial exploration work. A primary objective is to expand the sensor and autonomy capabilities our system to be more capable of science work. 
Integrate additional low cost vision capabilities and modes: Once we have completed our flight testing of the UAV with the secondary processor, we plan to add a gimbaled camera to the aircraft. This will allow random as well as direct observation of ground objects in flight. We would also like to add additional vision processing modes, to include imageprocessing algorithms that are of the most interest to science.

Provide additional autonomy software components. The Unix based PC 104 system was chosen in part because of the breadth of autonomy algorithms (planners, executives, system modules, data processing) that are available within the NASA and university robotic community. We would like to examine how these modules could be integrated and what affect they might have on our architecture.

Explore low cost coordinated image fusion. Based on our preliminary experience with aerial images, we would like to explore the coordination of imagery information through a secondary entity (for example, the location of an area of interest identified by a radio controlled aircraft or ATC rover surrogate relayed through a wireless link to the primary aircraft).

Expand the utility of deployed sensor pods: To include distributed sensor networks (multiple drop pods and locations); tailored emplacement of sensors (hillsides, canyon/valley walls), and different pod types: (ground penetrators, gliders, micro-rovers and "tetherbots").

\section{Field Science and Field Robotic Assistants.}

Equally important is to continue to expand our work with the science community in order to identify new ways in which aerial explorers can be used to enhance their work. There is promising follow-on research in that these bio-inspired mission scenario and autonomy concepts could be applied to robotic field assistants for geologists and biologists performing terrestrial field science in extreme environments ${ }^{10}$.

Imaging survey work is only one potential capability these aerial robotic field assistants could provide to the field researcher. More than one type of aerial vehicle configuration might be appropriate for conducting a field campaign. A whole new aerial vehicle design space might well open up for such robotic field assistants. Further, a "system of systems" of robotic devices (ground and air) and automated tools will likely be necessary. Some near term objectives would include:

Perform a quantitative assessment of various search and find strategies: Evaluate the utility of various algorithms by seeding multiple targets over an area or choosing different areas. This should lead to the ability to make quantifiable statements such as 3 of 4 targets identified within a rough terrain search area, in a fifteen minute search, with an Independent Random Walk strategy.)

Coordinate with a field astrobiologist to augment his or her field work. For example, acquire detailed mosaic mapping of rock formation faces for context, based on prior selection of a site

Apply the UAV as an augmentation to a ground system. For example, accompany a mobile research vehicle on a multiple day trek, performing aerial surveys at each key stopping point. The resulting aerial image mosaics would be deliverabie to trek organizers.

\section{CONCLLDING REMARKS}

Autonomous uninhabited aerial vehicles, acting as "aerial explorers," can potentially have a significant positive impact on NASA science missions. Whether for the purpose of acting as robotic field assistants for scientists conducting research in remote terrestrial extreme environments, or in support of planetary science missions, the development and use of aeria: explorers present a valuable opportunity to maximize scientific return on investment.

Key to the development of such aerial explorers are simple robust architectures for vehicle autonomy. Small autonomous aerial vehicles, particularly in the context of search and find missions, present unique challenges as compared to other robotic platforms/applications. The authors presented an outline of a "bio-inspired" architecture for autonomous aerial explorers, as well as summarized the development status of their effort. This architecture is heavily dependent upon defining flight "behaviors" actions and observations -- and effecting decisionmaking via concepts regarding robotic "ecology" (to model information flow/usage through discrete producer, consumer, and decomposer agents/processes) and "emotional holons" (to drive these processes)

Finally, representative simulation and flight demonstration results were presented. Flight 
demonstrations entailing three behaviors were conducted at Moffett Field airstrip at Ames Research Center. Additionally, an exploratory field demonstration was conducted at a remote Mars-analog site: Haughton Crater, Devon Island, Canada.

\section{ACKNOWLEDGMENTS}

The authors gratefully acknowledge the programmatic support and overall contributions of Dr. Steven Zometzer, Acting Deputy Director for at NASA Ames, Dr. Butler Hine, Manager of the NASA Intelligent Systems Program, and Mr. Edwin Aiken, Chief of the Army/NASA Rotorcraft Division. The authors also acknowledge the contributions of Dr. Pascal Lee of the SETI Institute for making possible the success of the autonomous aerial vehicle field demonstrations at Haughton Crater, Devon Island, NT, Canada. Finally, the authors also acknowledge the technical contributions of Dr. Steve Morris and other members of the staff of MLB, Inc., for the system integration and operation of the autonomous aerial vehicle.

\section{REFERENCES}

1. Aiken, E.W., Ormiston, R.A., and Young, L.A., "Future Directions in Rotorcraft Technology at Ames Research Center," $56^{\text {th }}$ Annual Forum of the American Helicopter Society, International, Virginia Beach, VA, May 2000.

2. Young, L.A., Chen, R.T.N., Aiken, E.W., and Briggs, G.A., "Design Opportunities and Challenges in the Development of Vertical Lift Planetary Aerial Vehicles," American Helicopter Society (AHS) Vertical Lift Aircraft Design Conference, San Francisco, CA, January 2000.

3. Pisanich, G., and Young, L.A., "An Aerobot Ecology," Robosphere 2002: Workshop on Self Sustaining Robotic Ecologies, NASA Ames Research Center, Moffett Field, CA, November 14$15,2002$.

4. Thakoor, S., Chahl, J., Srinivasan, M.V., Werblin, F., Young, L., Hine, B., Zornetzer, S., "Bioinspired Engineering of Exploration Systems for NASA and DoD", Artificial Life Journal, 2002, Vol. 8, Issue 4 , pages 357-369.

5. Plice, L., Pisanich, G., Lau, B., and Young, L., "Biologically Inspired 'Behavioral' Strategies for
Planning and Execution of Missions Using General Classes of Aerial Explorers", 2003 IEEE Aerospace Conference, Big Sky, MT, March 8-15, 2003.

6. Plice, L., "Robot Economy," Robosphere 2002: Workshop on Self-Sustaining Robotic Ecologies, NASA Ames Research Center, Moffett Field, CA, November 14-15, 2002.

7. Thakoor, S., "Bio-Inspired Engineering of Exploration Systems", Journal of Space Mission Architecture, Issue 2, Fall 2000, p 49-79.

8. Ippolito, C., Plice, L., and Pisanich, G., "Holarchical Systems and Emotional Holons: Biologically-Inspired System Designs for Control of Autonomous Aerial Vehicles," $7^{\text {th }}$ World Conference on Systematics and Cybemetics, Orlando, FL, July 2003.

9. Pisanich, G., Young, L.A., Ippolito, C., Lau, B., Plice, L., Sargent, R., and Lee, P., "Initial Efforts towards Mission-Representative Imaging Surveys from Aerial Explorers," International Society of Optical Engineering (SPIE) Electronic Imaging Conference, San Jose, CA, January 2004.

10. Young, L.A., Aiken, E.W., and Briggs, G.A., "Smart Rotorcraft Field Assistants for Terrestrial and Planetary Science," 2004 IEEE Aerospace Conference, Big Sky, MT, March 2004.

11. Lee, P., "Mars on Earth: The NASA HaughtonMars Project," Ad Astra: The Magazine of the National Space Society, Volume 14, Number 3, May/June 2002. 